\title{
Electroacupuncture Promotes the Survival of the Grafted Human MGE Neural Progenitors in Rats with Cerebral Ischemia by Promoting Angiogenesis and Inhibiting Inflammation
}

\author{
Juan Li $\mathbb{D},{ }^{1}$ Luting Chen $\mathbb{D},{ }^{2}$ Danping Li $\mathbb{D},{ }^{1}$ Min Lu $\mathbb{D},{ }^{1}$ Xiaolin Huang, ${ }^{1}$ Xiaohua Han $\mathbb{D},{ }^{1}$ \\ and Hong Chen (i) 1 \\ ${ }^{1}$ Department of Rehabilitation Medicine, Tongji Hospital, Tongji Medical College, Huazhong University of Science and Technology, \\ Wuhan, Hubei, China \\ ${ }^{2}$ Department of Rehabilitation Medicine, General Hospital of the Yangtze River Shipping, Wuhan, Hubei, China
}

Correspondence should be addressed to Xiaohua Han; hanxiao1470@hust.edu.cn and Hong Chen; chenhong1129@hust.edu.cn

Juan Li and Luting Chen contributed equally to this work.

Received 10 May 2021; Revised 11 July 2021; Accepted 6 September 2021; Published 7 October 2021

Academic Editor: Xi-Ze Jia

Copyright (c) 2021 Juan Li et al. This is an open access article distributed under the Creative Commons Attribution License, which permits unrestricted use, distribution, and reproduction in any medium, provided the original work is properly cited.

\begin{abstract}
Stem cells have the potential as a regenerative therapy for cerebral ischemia by improving functional outcomes. However, cell transplantation has some limitations, including a low rate of the grafted cell survival. There is still a major challenge of promoting the harmonious symbiosis between grafted cells and the host. Acupuncture can effectively improve the functional outcome after cerebral ischemia. The present study evaluated the therapeutic effects and explored the mechanism of combined medial ganglionic eminence (MGE) neural progenitors differentiated from human embryonic stem cells (hESCs) with electroacupuncture (EA) in a bilateral common carotid artery occlusion (2VO) rat model. The results showed that EA could promote the survival of the grafted MGE neural progenitors differentiated from hESCs and alleviate learning and memory impairment in rats with cerebral ischemia. This may have partially resulted from inhibited expression of TNF- $\alpha$ and IL- $1 \beta$ and increased vascular endothelial growth factor (VEGF) expression and blood vessel density in the hippocampus. Our findings indicated that EA could promote the survival of the grafted MGE neural progenitors and enhance transplantation therapy's efficacy by promoting angiogenesis and inhibiting inflammation.
\end{abstract}

\section{Introduction}

Ischemic cerebrovascular disease is a leading cause of mortality and disability worldwide after heart disease and cancer, lacking effective therapeutic methods [1]. In addition to causing hemiplegia, aphasia, swallowing disorders, etc., cerebral ischemia can also lead to a selective and delayed pyramidal neuronal death in the hippocampus and impair cognition function $[2,3]$. Rapid vascular recanalization is a relatively effective therapy. However, some patients cannot get timely access to effective treatment because the window for vascular recanalization is very short [4] and often results in intracranial hemorrhage [5]. Some drugs proven effective in animals could not reach desired therapeutic benefit in the clinic $[6,7]$.
Therefore, further investigation on effective treatments and interventions against cerebral ischemia is urgently needed.

Recently, different kinds of stem cells have been transplanted to treat central nervous system diseases, such as stroke [8], spinal cord injury [9, 10], and Alzheimer's disease (AD) [11]. MGE (medial ganglionic eminence) is a structure of the ventral forebrain during embryonic development. A study found that in the absence of NKX2.1 (the main marker of MGE), cholinergic septohippocampal projection neurons and large subsets of basal forebrain cholinergic neurons fail to develop, causing severe deficiencies in learning and memory [12]. MGE neural progenitors can be used as a cell source to treat learning and memory impairment. Cell therapy can supply a sufficient number of cells for 
transplantation, but there is a major challenge in promoting the harmonious symbiosis between grafted cells and the host. After a cerebral ischemic injury, most grafted cells die as a result of malignant changes in the ischemic focus and the surrounding microenvironment; for example, inflammation or immune response, trophic factor withdrawal, oxidative stress, excitotoxicity, hypoxia, and apoptosis [13-16]. Hence, to a certain extent, the unfriendly microenvironment restricts the effect of cell transplantation therapy. Therefore, improving microenvironment and promoting the survival of MGE neural progenitors may be one of the strategies to enhance the transplantation effect and improve the cognitive impairment after cerebral ischemia.

Electroacupuncture (EA) delivers electrical stimulation to acupuncture points through acupuncture needles. Several studies have shown that EA can effectively improve neural function recovery after cerebral ischemia. The potential mechanisms include prevention of inflammatory and oxidant stress [17], suppression of apoptosis [18], and promotion of angiogenesis [19]. Additionally, a systematic review and meta-analysis indicated that its mechanism positively correlates with endogenous neurogenesis, in which EA therapy can promote the migration and differentiation of neural stem cells (NSCs) [20]. Studies have confirmed the vital role of electroacupuncture in treating central nervous system diseases combined with stem cell transplantation [21, 22]. Therefore, EA could improve the host's brain's microenvironment with cerebral ischemia and promote the survival of grafted MGE neural progenitors to improve the cognitive impairment. This article will focus on promoting angiogenesis and inhibiting inflammation after cerebral ischemia to verify the above hypothesis.

\section{Materials and Methods}

2.1. Human Embryonic Stem Cell (hESC) Culture and Neuronal Differentiation. The hESCs (hM3Dq-KORD, Passages 25-50; WiCell Research Institute) were cultured on a feeder layer of irradiated mouse embryonic fibroblasts (MEFs) using hESC medium, which included DMEM/F12 (Hyclone, C11330500BT), 20\% knockout serum replacer (Gibco, 10828028), 1x GlutaMAX ${ }^{\mathrm{TM}}$ Supplement (Gibco, 35050061), 1x MEM Nonessential Amino Acid Solution Supplement (Gibco, 11140050), $0.1 \mathrm{mM} \beta$-mercaptoethanol (Sigma-Aldrich, M3148), and $8 \mathrm{ng} / \mathrm{mL}$ basic fibroblast growth factor (PeproTech, 10018B). The cells were expanded every six days.

MGE neural progenitors were differentiated by the dual SMAD inhibition differentiation protocol with MEFs as feeder layer, as described previously [23]. Briefly, the hESCs were cultured in neural differentiation medium, including 50\% DMEM/F12 (Gibco, 11330032), 50\% Neurobasal (Gibco, 21103049), 1x MEM Nonessential Amino Acid Solution Supplement, 1x N2 (Gibco, 17502048), $2 \mu \mathrm{M}$ SB431542 (Selleck, S1067), $2 \mu \mathrm{M}$ DMH1 (Selleck, S7146), and $2 \mu \mathrm{M}$ XAV939 (Stemgent, 040046) for seven days. The stem cells were differentiated into neuroepithelia in this process. Then, for ventralization, $0.5 \mu \mathrm{M}$ SAG (Sigma-Aldrich,
566660), a sonic hedgehog activator, was added at days 814 and the cells differentiated into NKX2.1 and Foxg1 coexpressing MGE neural progenitors. On day 14, the cells were digested and expanded as free-floating neural spheres for six days. On day 21, the neural spheres could be dissociated for transplantation or in vitro analysis. For in vitro analysis, the progenitor cells were spread onto polyornithine/laminincoated coverslips with neurobasal medium with BDNF (10 ng/mL; PeproTech), GDNF (10 ng/mL; PeproTech), IGF1 (10 ng/mL; PeproTech), and cAMP ( $1 \mu \mathrm{M}$, SigmaAldrich) (Figure 1).

2.2. Animals and Bilateral Common Carotid Artery Occlusion (2VO) Model. SPF-grade adult male SpragueDawley (SD) rats, weighing 250-280 g, were purchased from the Center of Experimental Animals, Tongji Medical College, Huazhong University of Science and Technology, Wuhan, China. All the procedures were approved by the Animal Care and Use Committee of the Tongji Medical College, Huazhong University of Science and Technology, Wuhan, China. SD rats were housed at $25 \pm 2^{\circ} \mathrm{C}$ under a $12 \mathrm{~h}$ light/dark cycle with food and water ad libitum. The bilateral common carotid artery occlusion (2VO) model was generated as described previously [24]. Animals were divided randomly into five groups: (1) sham operation group (sham), (2) $2 \mathrm{VO}$ group (2VO), (3) $2 \mathrm{VO}$ combined with culture medium transplantation group (2VO+Vehicle), (4) $2 \mathrm{VO}$ combined with MGE neural progenitor transplantation group $(2 \mathrm{VO}+\mathrm{Cell})$, and (5) $2 \mathrm{VO}$ combined with MGE neural progenitor transplantation and EA group $(2 \mathrm{VO}+\mathrm{Cell}+\mathrm{EA})$.

2.3. Electroacupuncture Therapy. The rats in the $2 \mathrm{VO}+\mathrm{Cell}$ + EA group received EA treatment. The location of the "Baihui" (GV20) and "Dazhui" (GV14) acupoints was described previously [24] (Figure 2(a)). Acupuncture needles $(0.3 \mathrm{~mm}$ diameter) were horizontally inserted at a depth of $10 \mathrm{~mm}$ into the GV20 and perpendicularly at $5 \mathrm{~mm}$ into GV14. Then, the electrical stimulation was delivered using a G6805-II electroacupuncture therapeutic apparatus (Shanghai Medical Electronic Apparatus Co., China), with a continuous current at $2 \mathrm{~Hz}$ for $20 \mathrm{~min}$ daily. The stimulation intensity was set according to the visible light facial muscle twitching. The entire treatment period was seven days starting from the first day after $2 \mathrm{VO}$ to the cell transplantation day (Figure 2(b)).

2.4. Cell Transplantation. Cell transplantation was performed seven days after $2 \mathrm{VO}$ as described previously [25]. In brief, MGE neural progenitors were dissociated with Accutase and prepared at approximately $1 \times 10^{5}$ cells $/ \mu \mathrm{L}$ in the medium. The rats were anesthetized and fixed on the stereotaxic device. Next, the skull was exposed, and a small hole was drilled on per hemisphere. A total of $2.5 \mu \mathrm{L}$ cell suspension was transplanted into per side of hippocampus site (coordinates: anterior-posterior $(\mathrm{AP})=-4.0 \mathrm{~mm}$, mediallateral $(\mathrm{ML})= \pm 3.0 \mathrm{~mm}$, and dorsal-ventral $(\mathrm{DV})=-3 \mathrm{~mm}$; 0 reference point for $\mathrm{AP}$ and $\mathrm{ML}$ at bregma, 0 reference point for DV at skull surface at the target site), according 


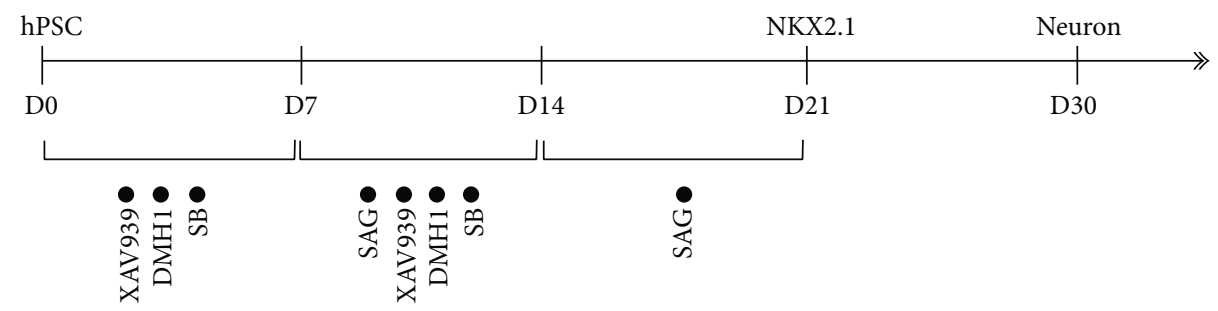

(a)
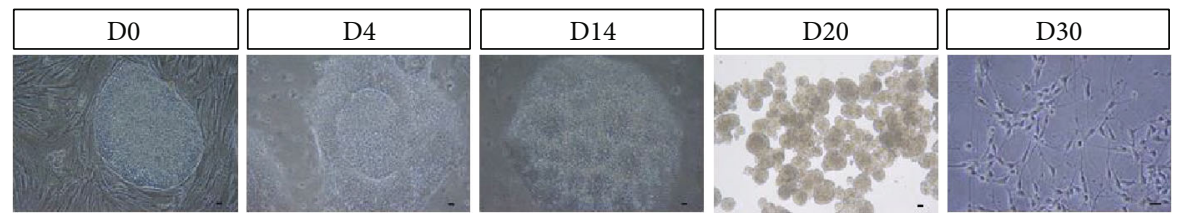

(b)

FIGURE 1: Differentiation of hESCs into MGE neural progenitors in vitro. (a) Flowchart of differentiation induced by the dual SMAD inhibition differentiation method. (b) Cell morphology at different stages of differentiation. Scale bars $=50 \mu \mathrm{m}$.

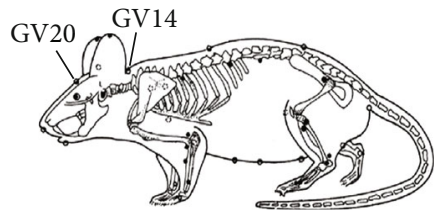

(a)

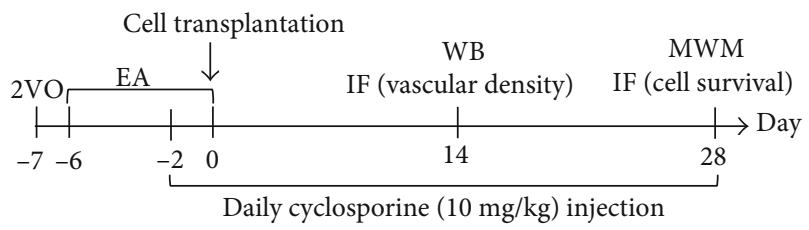

(b)

Figure 2: (a) Schematic diagram of GV20 and GV14 acupoints in the rat. (b) Flowchart of the experimental design.

to the stereotaxic coordinates given in the rat atlas of Paxinos and Watson [26]. The injection time was continuous $5 \mathrm{~min}$, and the needle was left for $5 \mathrm{~min}$ before removal to prevent cells from overflowing the needle track. All rats were injected subcutaneously with $10 \mathrm{mg} / \mathrm{kg}$ cyclosporine A, two days before transplantation and subsequently daily.

2.5. Laser Doppler Flowmetry. The cortical cerebral blood flow (CBF) was assessed using a laser Doppler flowmetry (MoorVMS, England). Rats were anesthetized and placed in a stereotactic apparatus. A burr hole ( $3 \mathrm{~mm}$ in diameter) was made through a midline scalp incision, with an electric drill on the right frontoparietal region (anterior-posterior ( $\mathrm{AP})=-1.0 \mathrm{~mm}$, medial-lateral $(\mathrm{ML})=-5.0 \mathrm{~mm})$ to set a placement device for a contact probe. The CBF was quantified before and after the ligation of the common carotid arteries. Calculated perfusion was expressed as a percentage ratio of postischemic to the preischemic brain to account for variables.

2.6. Tissue Processing. Rats were deeply anesthetized with $10 \%$ chloral hydrate $(3 \mathrm{~mL} / \mathrm{kg})$ and perfused with $4 \%$ paraformaldehyde (PFA). The brains were quickly dissected out and soaked in 4\% PFA for at least $12 \mathrm{~h}$. The rat brain tissues were subjected to gradient dehydration in $20 \%$ and $30 \%$ sucrose sequentially. The brain specimens containing transplantation sites (from 3.0 to $5.0 \mathrm{~mm}$ behind the bregma) were embedded in optimal cutting temperature compound and cut into $30 \mu \mathrm{m}$ coronal tissue sections with a frozen tissue slicer. The tissue sections were collected further in tissue cryopreservation fluid for immunohistochemical analysis.

2.7. Immunofluorescence Staining. Immunofluorescence staining was performed in vitro on coverslip cultures or frozen brain tissue sections as previously described [27]. Briefly, cells were fixed in 4\% PFA for $20 \mathrm{~min}$ (frozen tissue sections did not require this step), washed with phosphate-buffered saline (PBS), and then blocked by QuickBlock ${ }^{\mathrm{TM}}$ Blocking Buffer for Immunol Staining (Beyotime, P0260) for $30 \mathrm{~min}$ at room temperature. The primary antibodies were added and incubated overnight at $4^{\circ} \mathrm{C}$. Primary antibodies included mouse anti-Nestin (Millipore, MAB5326, 1:1000), rabbit anti-PAX6 (BioLegend, 901301, 1:1000), goat anti-SOX1 (R\&D Systems, AF3369, 1:1000), rat anti-HOXB4 (DSHB, RRID: AB-2119288, 1:100), rabbit anti-Foxg1 (Abcam, Ab18259, 1:500), mouse anti-NKX2.1 (Millipore, MAB5460, 1:500), rabbit anti-GABA (Sigma-Aldrich, A2052, 1:1000), goat anti-CHAT (Millipore, AB144P, $1: 300$ ), mouse anti-neuronal class III $\beta$-tubulin (Tuj1; BioLegend, 801201, 1:2000), mouse anti-microtubuleassociated protein-2 (Map2; Sigma-Aldrich, M1406, $1: 1000)$, mouse anti-human nuclei (HuNu; Millipore, MAB1281, 1:500), goat anti-mCherry (Biorbyt, RRID: AB2687829), and rabbit anti-NeuN (Millipore, ABN78, $1: 1000)$. The corresponding fluorescent-conjugated secondary antibodies were applied for $1 \mathrm{~h}$, and the nuclei were stained with DAPI (Boster, AR1177). 
2.8. Vascular Density Analysis. Vascular density in the hippocampus was evaluated with immunofluorescence staining on the $14^{\text {th }}$ day after grafting. Coronal brain sections $(30 \mu \mathrm{m})$ on every six sections were used for vascular density analysis. The stained vasculature was observed within the hippocampus using an Olympus FV1000 confocal microscope (Olympus, Japan, $\times 10$ objective). A lectin-positive vessel separated from adjacent vessels was counted as one vessel. The number of these vessels was added to the number of vascular branch points (number of vessel bifurcations) to obtain the total number of vessels, as described previously [28]. Five fields for each section, randomly distributed, were imaged for statistical analysis, with at least three independent replication tests. An investigator blinded to the group manually quantified vascular density, and the data were expressed as the number of stained vessels per $0.36 \mathrm{~mm}^{2}$ under $\times 10$ objective.

2.9. Microscopical Analysis and Cellular Quantification. As described previously [25], all nuclei of grafted cells were identified in the presence of the human-specific nuclear marker $(\mathrm{HuNu})$ immunostaining. $\mathrm{HuNu}^{+} / \mathrm{DAPI}^{+}$doublelabeled cell counting was performed on every six sections on the $28^{\text {th }}$ day after grafting [29], and images were captured using an Olympus FV1000 confocal microscope (Olympus, Japan, $\times 40$ objective). The numbers of $\mathrm{HuNu}^{+} / \mathrm{DAPI}^{+}$ double-labeled cells were counted on the images by an investigator blinded to the group using ImageJ software (NIH, Bethesda, Maryland, USA), and the data were expressed as the number of positive cells per area under $\times 40$ objective. Five areas were randomly selected for each section, with at least three independent replication tests.

2.10. Western Blot Analysis. Under deep anesthesia, hippocampus brain tissue, including the grafting area, was immediately dissected on ice. After centrifugation at $12,000 \mathrm{rpm}$ for $15 \mathrm{~min}$, total protein was isolated by homogenization, and the supernatant was collected. The protein concentrations were estimated by BCA assay. The total protein was separated using sodium dodecyl sulfate-polyacrylamide gel electrophoresis (SDS-PAGE). After electrophoresis, it was transferred onto polyvinylidene difluoride (PVDF) membranes, blocked in milk, and incubated at $4^{\circ} \mathrm{C}$ overnight with primary antibodies: rabbit anti-VEGF (Affinity, AF5131, $1: 1000)$, rabbit anti-TNF- $\alpha$ (CST, 8184S, $1: 1000)$, and rabbit anti-IL-1 $\beta$ (CST, 12703S, $1: 1000$ ), followed by incubation with appropriate horseradish peroxidase-conjugated secondary antibodies. Blots were detected using the ECL Western Blotting Substrate, and the intensity of the protein band was quantified using Gel-Pro Analyzer 4.0 software (Media Cybernetics, USA).

2.11. Morris Water Maze Task. Morris water maze (MWM) task was used to evaluate hippocampus-dependent spatial learning and memory. Briefly, a circular water tank $(150 \mathrm{~cm}$ in diameter and $50 \mathrm{~cm}$ in deep) was filled with water $\left(23 \pm 2^{\circ} \mathrm{C}\right)$ to a depth of $21 \mathrm{~cm}$. A circular submerged platform $(15 \mathrm{~cm}$ in diameter and $20 \mathrm{~cm}$ in height) was placed underwater and in the target quadrant center. Several visual cues were displayed on the wall of the test room. In the place navigation phase, rats were subjected to four trials per day for four consecutive days. Each trial lasted either until the rat found the platform or for $60 \mathrm{~s}$. The starting point was changed for every trial. The rats were allowed to rest on the platform for $20 \mathrm{~s}$ after each trial. On the $5^{\text {th }}$ day, the platform was removed for a $60 \mathrm{~s}$ spatial probe trial to test rats' spatial memory. The latency to find the submerged platform, the dwell time in the target quadrant, and the swimming paths were recorded automatically using a computerbased image analyzer MWM tracking system MT-200 (Chengdu Technology \& Market Co., Ltd., Chengdu, Sichuan Province, China).

2.12. Statistical Analysis. SPSS 22.0 software (IBM Corporation, Somers, New York, USA) was used for statistical analysis. To assess the normal distribution of data, the ShapiroWilk test was employed $(P>0.05)$. Data were analyzed for multiple comparisons by one-way analysis of variance (ANOVA) followed by Tukey's multiple comparison tests among different groups. When comparing two groups at two time points (CBF values), two-way analysis of variance (ANOVA) was implemented. When comparing two groups at the same time point, Student's $t$-test was implemented. All data were expressed as mean \pm standard error of the mean (SEM). $P$ value $<0.05$ was considered statistically significant.

\section{Results}

3.1. Human MGE Neural Progenitors Were Efficiently Generated from hESCs In Vitro. During the culture process, small molecules SB431542, DMH1, XAV939, and SAG were applied to promote hESC differentiation into neural progenitor cells. Morphologically, neural tube structures appeared on the $4^{\text {th }}$ day of differentiation, and several rosettes, the typical structure of the neuroepithelium, appeared on the $14^{\text {th }}$ day. After being expanded as free-floating neural spheres, MGE neural progenitors were obtained on the $21^{\text {st }}$ day. The cells were then adherently cultured at this stage, and neurons were obtained after ten days (Figure 1(b)).

In the presence of several small-molecule inhibitors, neuroepithelium marker PAX6 and NSC marker Nestin began to appear on the $4^{\text {th }}$ day (Figure $3(\mathrm{a})$ ). On the $10^{\text {th }}$ day, another marker of the neuroepithelium, SOX1, was observed (Figure 3(b)). Additionally, the forebrain marker, FOXG1, but no spinal cord marker, HOXB4, was observed (Figure 3(c)). Meanwhile, the cells colabeling MGE marker NKX2.1 and FOXG1 appeared on the $14^{\text {th }}$ day (Figure 3(d)) and reached a peak on the $21^{\text {st }}$ day. MGE neural progenitors eventually differentiated into GABAergic and cholinergic neurons on the $49^{\text {th }}$ day in vitro (Figures 3(e) and $3(\mathrm{f})$ ).

3.2. The 2VO Model Showed a Decrease in Cerebral Blood Flow (CBF). The pre- and postoperative blood flow was monitored in rats of the sham and $2 \mathrm{VO}$ groups with laser Doppler. Before the operation, the mean baseline CBF values in the sham and $2 \mathrm{VO}$ groups were $171.9 \pm 6.07(\mathrm{PU})$ and 


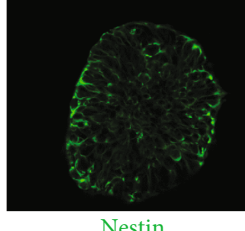

Nestin

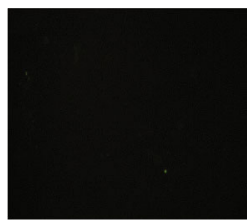

HOXB4

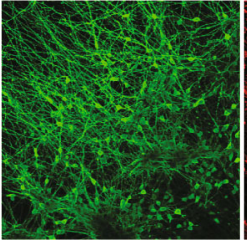

GABA

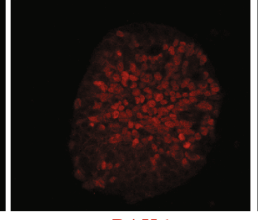

PAX

(a)

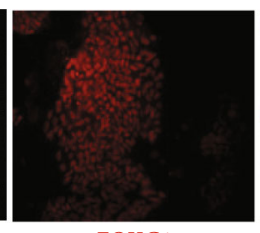

FOXG1

(c)

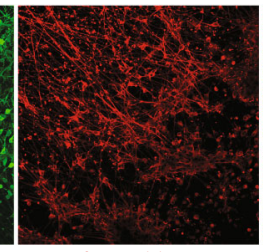

$\beta$ III-tublin

(e)

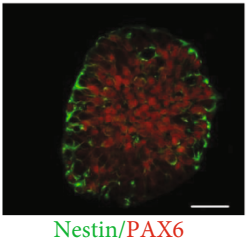

Nestin/PAX6

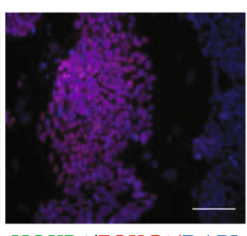

HOXB4/FOXG1/DAPI

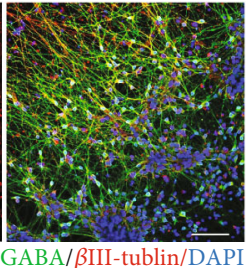

GABA/ $\beta$ III-tublin/DAP

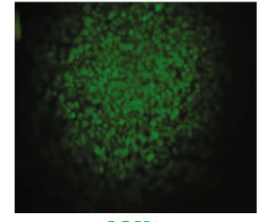

SOX1

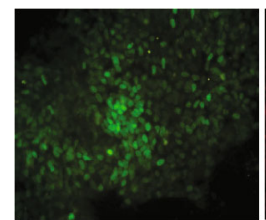

NKX2.1

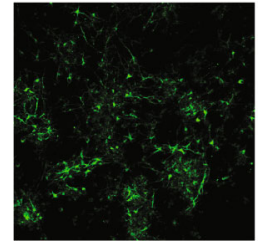

CHAT

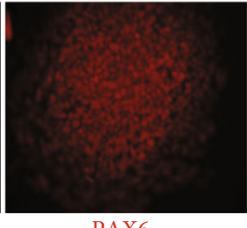

PAX6

(b)

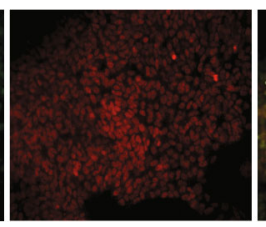

FOXG1

(d)

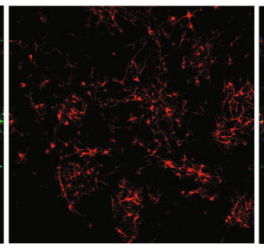

MAP2

(f)

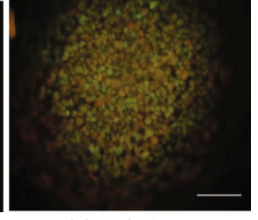

SOX1/PAX6

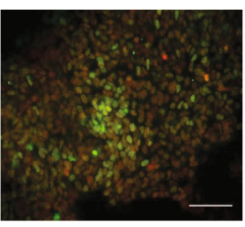

NKX2.1/FOXG1

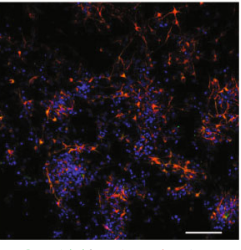

CHAT/MAP2/DAPI

FIGURE 3: Generation of neural progenitors for transplantation and the neural markers of MGE neural progenitors during the process of differentiation. (a) Representative images were immunoreactive for neuroepithelium marker PAX6 and NSC marker Nestin on day 4. (b) Coimmunostaining SOX1 and PAX6 at day 10. (c) Differentiated hESCs expressed forebrain marker, FOXG1, but no spinal cord marker, HOXB4. (d) Coexpressing MGE neural progenitor marker NKX2.1 and FOXG1 on day 14. By 49 days in culture, neurons were positive for (e) GABA and (f) CHAT. Scale bars $=50 \mu \mathrm{m}$.

$173.4 \pm 5.26(\mathrm{PU})$, respectively, with no significant difference. The $\mathrm{CBF}$ value in the $2 \mathrm{VO}$ group decreased to 54.9 $\pm 6.45(\mathrm{PU})$, reaching the lowest within $5 \mathrm{~min}$ after the operation, and then maintained a low blood flow state, which indicated that $2 \mathrm{VO}$ rats suffered from persistent cerebral hypoperfusion (Figure 4).

\subsection{EA Inhibited Inflammation of the Hippocampus in Rats} of the 2VO+Cell Group. Earlier studies suggested that postischemic inflammation plays a vital role in various stages of cerebral ischemic injury. Activated microglia leads to inflammasome-mediated interleukin- (IL-) $1 \beta$ release, as well as tumor necrosis factor (TNF) production, which feedbacks into the inflammatory cascade by inducing cytokine and chemokine production in endothelial cells and astrocytes [30]. The inflammation leads to the deterioration of the local microenvironment, which is toxic to the host neural cells and is not conducive to the survival of exogenous grafted cells. It was reported that chronic cerebral hypoperfusion-induced cognitive impairment is associated highly with inflammation [31]. Therefore, proinflammatory cytokines were detected by Western blot analysis in this study to evaluate whether EA can inhibit inflammation, promote grafted cell survival, and alleviate cognition impairment. 2VO-induced inflammatory response in rats' hippocampus was demonstrated by the increased immunoreactivity of TNF- $\alpha$ and IL- $1 \beta$ compared to the sham group
$(P<0.001)$. In the $2 \mathrm{VO}+\mathrm{Cell}+\mathrm{EA}$ group, immunoreactivity of TNF- $\alpha$ and IL- $1 \beta$ in the hippocampus decreased significantly compared with that of the $2 \mathrm{VO}+$ Cell group $(P=0.008$ and $P=0.029)$, indicating that EA inhibited inflammation response of the hippocampus in rats of the 2VO+Cell group (Figure 5).

3.4. EA Promoted Angiogenesis of the Hippocampus in Rats of the 2VO+Cell Group. The key regulator of vascular development and homeostasis is the vascular endothelial growth factor (VEGF) [32]. In our study, Western blot analysis revealed a significant difference in VEGF protein expression in the hippocampus among the five groups. Two-vessel occlusion induced elevated VEGF protein expression in the hippocampus. Rats in the 2VO+Cell and $2 \mathrm{VO}+\mathrm{Cell}+\mathrm{EA}$ groups showed increased VEGF expression compared with the $2 \mathrm{VO}$ group at day 14 after transplantation. Compared with the 2VO+Cell group, VEGF protein expression was significantly higher in the $2 \mathrm{VO}+\mathrm{Cell}+\mathrm{EA}$ group rats $(P<0.001)$. Additionally, the vascular density of the cell grafting area in the hippocampus was detected by immunofluorescence staining. Vascular density in the 2VO+Cell $+\mathrm{EA}$ group rats $(52.25 \pm 6.50)$ was significantly higher than that in the $2 \mathrm{VO}+$ Cell group $(32.75 \pm 3.25)$ on day 14 after cell grafting $(P=0.017)$ (Figure 6). The above results indicated that $\mathrm{EA}$ promoted angiogenesis of the hippocampus in $2 \mathrm{VO}+$ Cell rats. 


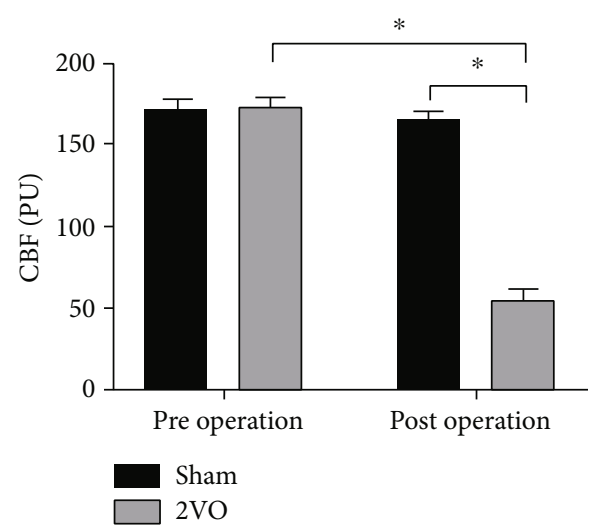

(a)

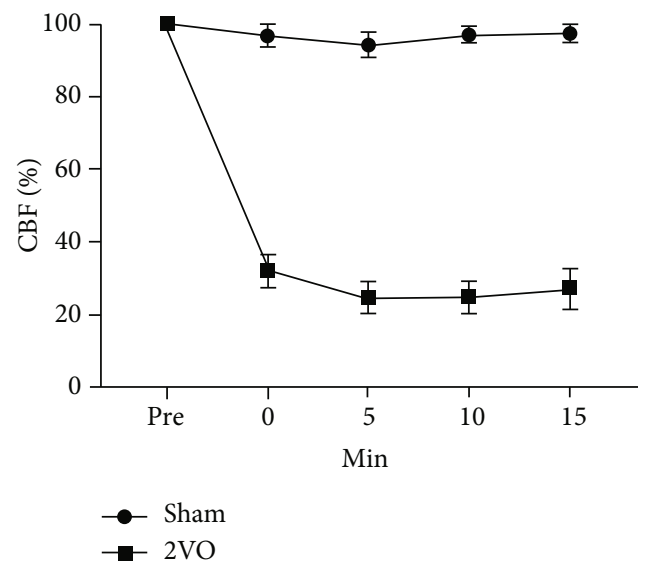

(b)

FIGURE 4: Laser Doppler monitoring of CBF in rats of the sham and 2VO groups. (a) Preoperative and postoperative blood flow values of the sham and 2VO group analysis of variance (two-way ANOVA, $F=275.036, P<0.001$ ). (b) The ratio of postischemic to preischemic CBF changes within $0-15$ min before and after surgery. Values are mean \pm SEM $\left(N=5\right.$ rats/per group). ${ }^{*} P<0.05$.

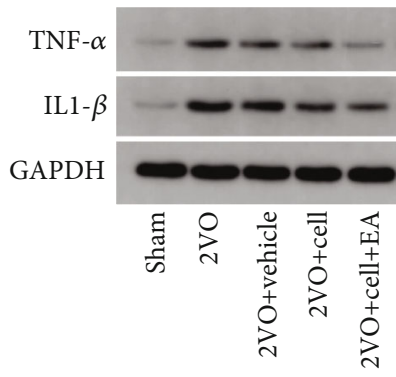

(a)

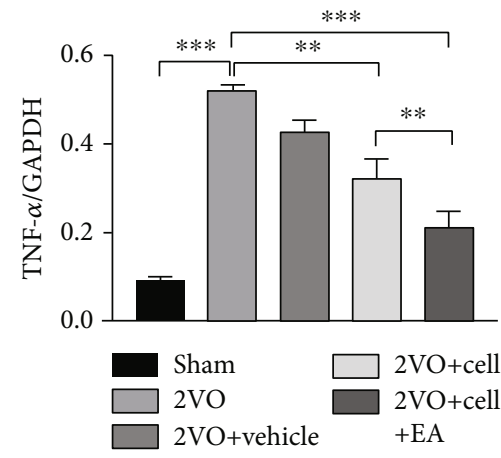

(b)

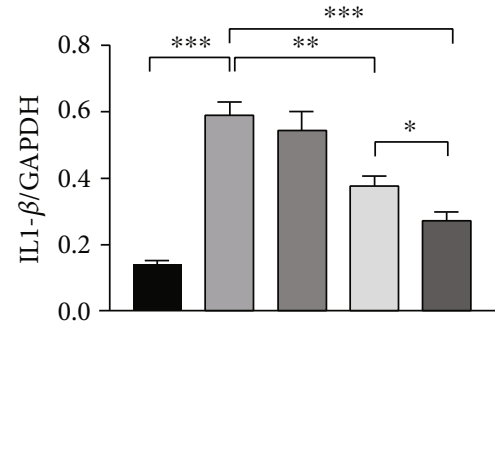

(c)

FIGURE 5: Effects of MGE neural progenitors and EA treatment on the expression of TNF- $\alpha$ and IL- $1 \beta$ in the hippocampus were evaluated by Western blot 14 days after grafting. (a) Gel electrophoresis of TNF- $\alpha$ and IL-1 $\beta$. (b, c) Densitometric analysis of TNF- $\alpha$ (one-way ANOVA, $F=91.711, P<0.001$ ) and IL-1 $\beta$ (one-way ANOVA, $F=57.059, P<0.001)$. Values are mean \pm SEM $\left(N=5\right.$ rats/per group). ${ }^{*} P$ $<0.05,{ }^{* *} P<0.01$, and ${ }^{* * *} P<0.001$.

3.5. EA Promoted the Survival of the Grafted MGE Neural Progenitors in the Hippocampus. To better trace the grafted cells, hESCs modified by mCherry at AAVS1 site were used to differentiate into MGE neural progenitors for transplantation. Four weeks after transplantation, a large number of cells comarked with $\mathrm{HuNu}$ and mCherry were observed at the grafting area, indicating that grafted MGE neural progenitors differentiated from hESCs still survived in rats (Figure 7(a)). Some grafted MGE neural progenitors differentiated into neurons, weakly expressing NeuN (Figure 7(b)). Further analysis revealed that much more $\mathrm{HuNu}^{+}$cells survived in the $2 \mathrm{VO}$ + Cell+EA group $(1151 \pm 260)$ than in the $2 \mathrm{VO}+$ Cell group $(540 \pm 123)$ in the grafting area $(P=0.021)$, indicating that EA promoted the survival of the grafted MGE neural progenitors in the hippocampus (Figures 7(c) and 7(d)).

3.6. MGE Neural Progenitor Transplantation Alleviated Learning and Memory Impairment in 2VO Rats and EA Enhanced Transplantation Therapy's Efficacy. Four weeks after transplantation, the MWM task was used to assess the learning and memory ability of rats in each group, including spatial learning (Figure 8(a), A-E) and spatial memory test (Figure 8(a), F-J). After four days of spatial learning training, the escape latencies of rats in the $2 \mathrm{VO}$ group $(47.2 \pm 4.5 \mathrm{~s})$ and the $2 \mathrm{VO}+\mathrm{Vehicle}$ group $(46.4 \pm 5.4 \mathrm{~s})$ were significantly longer than those in the sham group $(23.7 \pm 1.9 \mathrm{~s})(P<0.001)$. Compared with rats in the $2 \mathrm{VO}$ group, rats in the $2 \mathrm{VO}+$ Cell group showed a reduction in the escape latency $(36.5 \pm 9.5 \mathrm{~s}, P<0.05)$ (Figure 8(b)) and showed increased time spent in the target quadrant $(14.2 \pm 3.9 \mathrm{~s}, P=0.036)$ (Figure $8(\mathrm{c}))$, while the frequency of crossing in the platform was not different $(1.4 \pm 0.55, P=0.759)$ (Figure $8(\mathrm{~d}))$. Compared with the $2 \mathrm{VO}+$ Cell group, the escape latency of rats in the $2 \mathrm{VO}$ + Cell+EA group was shortened without significant difference $(32.0 \pm 2.2 \mathrm{~s}, P=0.786)$ (Figure $8(\mathrm{~b}))$. However, rats in the $2 \mathrm{VO}+\mathrm{Cell}+\mathrm{EA}$ group showed increased time spent in the target quadrant $(21.6 \pm 3.5 \mathrm{~s}, P=0.019)$ and increased 


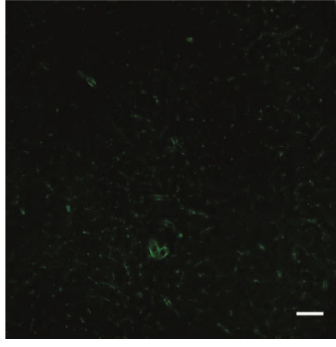

2VO+cell

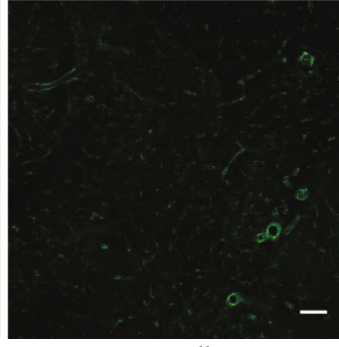

$2 \mathrm{VO}+\mathrm{cell}+\mathrm{EA}$

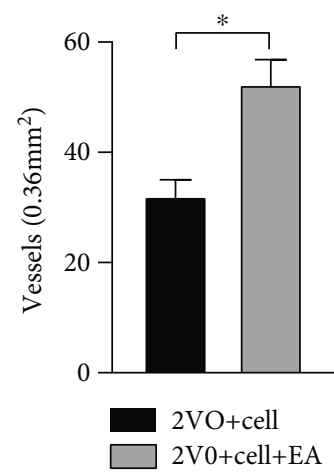

(b) (a)

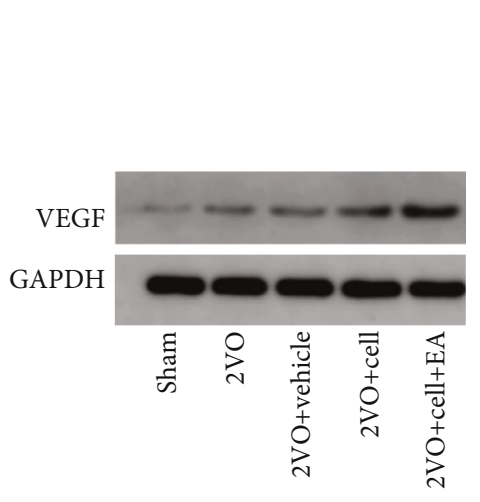

(c)

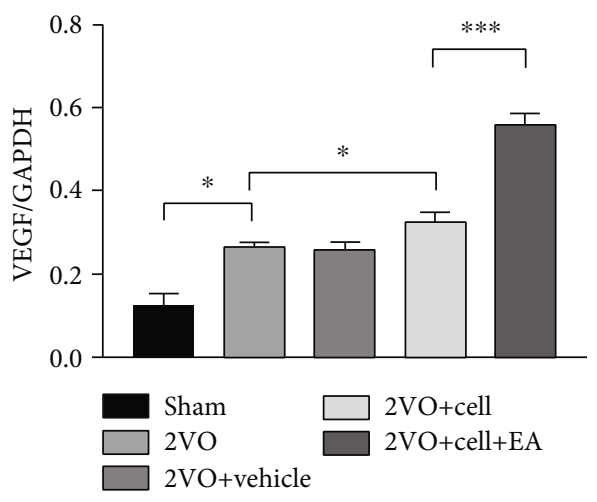

(d)

FIGURE 6: EA facilitates hippocampal angiogenesis in 2VO+Cell rats. (a) Immunofluorescence staining of blood vessels (green) in the hippocampus on the $14^{\text {th }}$ day after grafting. Scale bar $=100 \mu \mathrm{m}$. (b) Quantification of vascular density in the 2VO+Cell and 2VO+Cell +EA groups. (c) Representative Western blots of VEGF on the $14^{\text {th }}$ day after grafting. (d) The densitometric analysis of VEGF level detected from the hippocampus in each group (one-way ANOVA, $F=13.574, P<0.001$ ). Values are mean \pm SEM $(N=5$ rats/per group). ${ }^{*} P<0.05$ and ${ }^{* * *} P<0.001$.

frequency of crossing the platform $(2.8 \pm 0.83, P=0.047)$ compared with the $2 \mathrm{VO}+$ Cell group (Figures $8(\mathrm{c})$ and $8(\mathrm{~d}))$. There were no significant differences between rats in the $2 \mathrm{VO}$ and $2 \mathrm{VO}+\mathrm{Vehicle}$ groups in escape latency, time spent in the target quadrant, and the frequency of crossing the platform. The above results of the MWM task indicated that MGE neural progenitor transplantation alleviated learning and memory impairment in $2 \mathrm{VO}$ rats, and EA enhanced transplantation therapy's efficacy.

\section{Discussion}

Some neurological diseases are closely related to different types of neuronal damage, reduction, and death. For example, cognitive dysfunction in cerebral ischemia is related to cholinergic neuron loss in the hippocampus [33, 34]. Recently, with the development of differentiation technology, human pluripotent stem cells, including human embryonic stem cells (hESC) and human-induced pluripotent stem cells (hiPSC), can be induced to differentiate into specific neurons and glial cells in vitro, which provide a good source for cell transplantation therapy for these neurological diseases [35].

MGE (medial ganglionic eminence) is a structure of the ventral forebrain during embryonic development, hav- ing the main marker, NKX2.1. NKX2.1 is very important for the development of embryonic septal neuroepithelium and cholinergic neurons in the basal forebrain and the hippocampal cholinergic projection system. A study found that in the absence of NKX2.1, cholinergic septohippocampal projection neurons and large subsets of basal forebrain cholinergic neurons fail to develop, causing alterations in the hippocampal theta rhythms and severe deficiencies in learning and memory [12]. MGE neural progenitors are mainly differentiated into GABAergic neurons and cholinergic neurons in vitro, used as a cell source to treat learning and memory impairment. In this study, human embryonic stem cells were used to differentiate into MGE neural progenitors by combining induction using different small molecules for subsequent cell transplantation treatment. In the in vitro cell differentiation stage, a large number of NKX2.1-positive cells were observed on the 21st day. After another 28 days, the differentiated cells expressed GABA and CHAT, suggesting that the MGE neural progenitors obtained according to our differentiation protocol can further differentiate into GABAergic and cholinergic neurons in vitro. It thus lays the foundation for MGE neural progenitors grafting to improve the cognitive impairment caused by cerebral hypoperfusion in rats. 

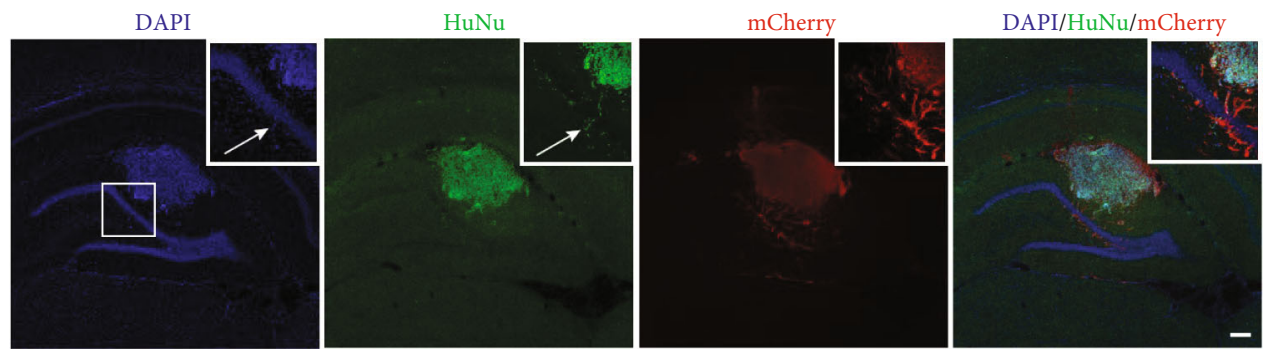

(a)
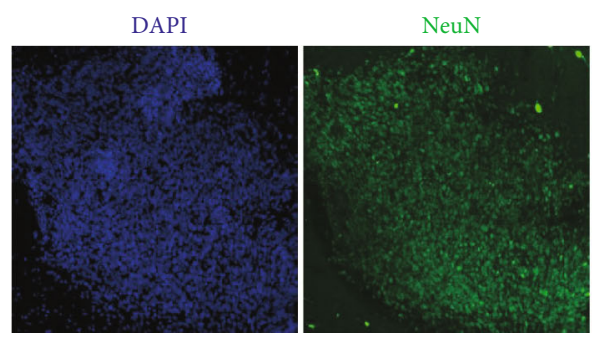

mCherry

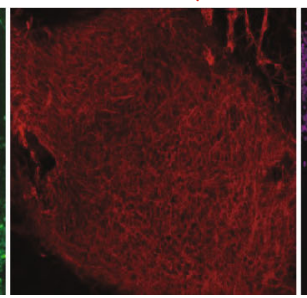

$\mathrm{HuNu}$

DAPI/NeuN/mCherry/HuNu

(b)

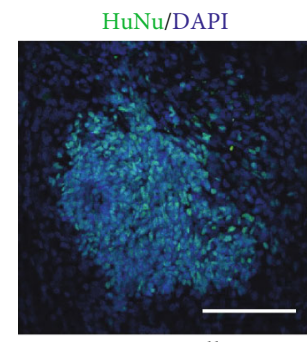

$2 \mathrm{VO}+$ cell

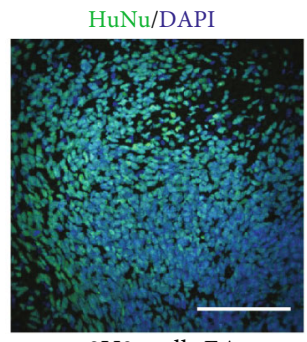

2V0+cell+EA
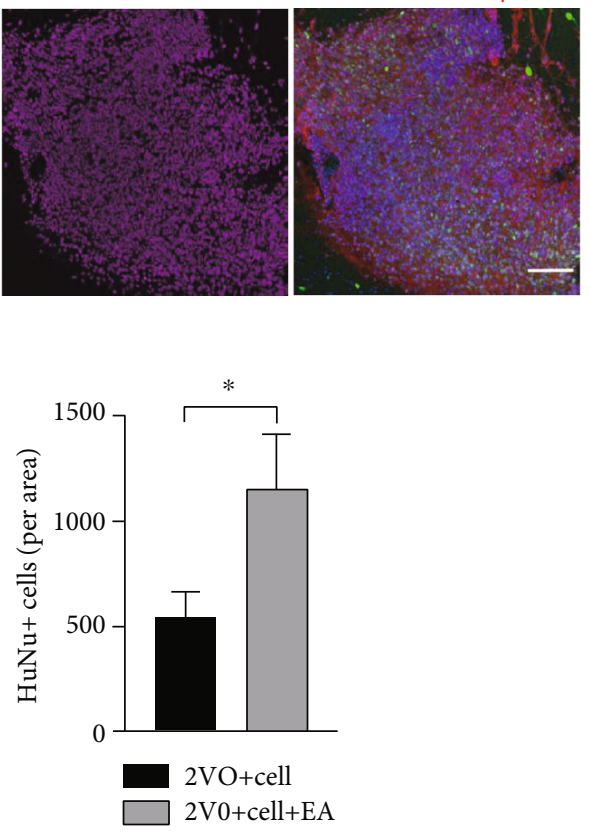

(d)

Figure 7: Effect of EA treatment on the survival of the grafted MGE neural progenitors in the hippocampus. (a) Fluorescence photomicrographs of $\mathrm{HuNu}^{+} / \mathrm{mCherry}^{+}$-positive cells from the $2 \mathrm{VO}+\mathrm{Cell}+\mathrm{EA}$ group. Scale bar $=200 \mu \mathrm{m}$. (b) Some $\mathrm{HuNu}^{+} / \mathrm{mCherry}^{+}-$ positive cells from the $2 \mathrm{VO}+\mathrm{Cell}+\mathrm{EA}$ group weakly expressed NeuN. Scale bar $=100 \mu \mathrm{m}$. (c) Fluorescence photomicrographs of HuNu ${ }^{+}-$ positive cells from the $2 \mathrm{VO}+\mathrm{Cell}$ and $2 \mathrm{VO}+\mathrm{Cell}+\mathrm{EA}$ groups. Scale bar $=100 \mu \mathrm{m}$. (d) Quantification of $\mathrm{HuNu}^{+}$-positive cells in the $2 \mathrm{VO}$ + Cell and $2 \mathrm{VO}+$ Cell + EA groups. Values are mean \pm SEM $(N=5$ rats/per group $) .{ }^{*} P<0.05$.

Neural stem cell transplantation can replace lost neurons and improve functional deficits because it has the inherent ability to differentiate into various cell phenotypes. Therefore, currently, cell transplantation has growing potential. However, there is still a long way to go, and some obstacles must be overcome before cell transplantation therapy can be actively used in the clinic. One of the major problems with cell transplantation is the low survival rate of grafted cells (5-20\%). Most cell death occurs during the first few days after transplantation as a result of inflammation or immune response, trophic factor withdrawal, oxidative stress, excitotoxicity, hypoxia, and apoptosis [13-16]. Thus, for successful transplantation, improving the ischemic and hypoxic state of the hippocampus and inhibiting the inflammation in the grafted area, thereby improving the survival environment of grafted cells, may be an important strategy to improve the survival rate of grafted cells.

As an important treatment of Traditional Chinese Medicine, EA delivers electrical stimulation to acupoints through acupuncture needles. It has been recommended as a complementary therapy in stroke rehabilitation in Asian and Western countries. An increasing number of studies have shown that acupuncture can effectively improve the functional recovery of neurons after cerebral ischemia [36,37]. The potential mechanisms include preventing inflammatory and oxidant stress, suppressing apoptosis, promoting angiogenesis, and endogenous neural regeneration. Since EA can promote angiogenesis and anti-inflammatory, it was combined with cell transplantation to study the survival of grafted cells in this study. Our findings showed that the $2 \mathrm{VO}$ model increased the expression of inflammatory factors, TNF- $\alpha$ and IL- $1 \beta$, in the hippocampus, and the hypoperfusion produced by $2 \mathrm{VO}$ induced a slight increase in VEGF protein, consistent with the results of other studies $[17,28]$. Compared with the $2 \mathrm{VO}+$ Cell group, EA inhibited TNF- $\alpha$ and IL- $1 \beta$ expression and increased the expression of VEGF protein and blood vessel density in the hippocampus of the $2 \mathrm{VO}+\mathrm{Cell}+\mathrm{EA}$ group. In the grafting area, several 


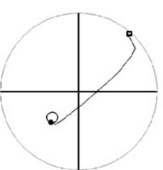

(A)

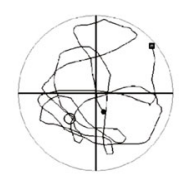

(F)

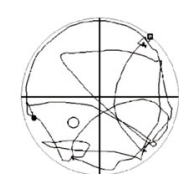

(B)

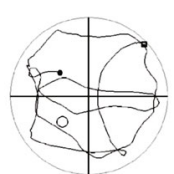

(C)

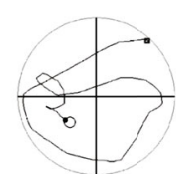

(D)

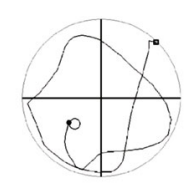

(E)

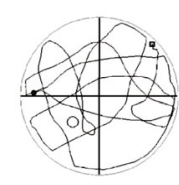

(G)

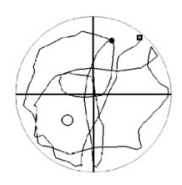

(H)

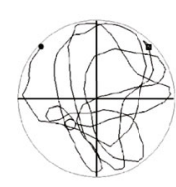

(I)

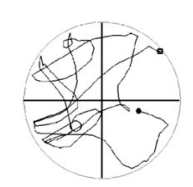

(J)

(a)

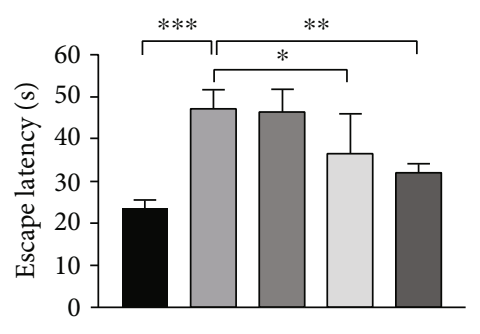

(b)

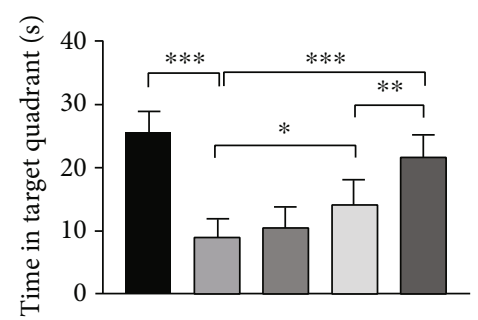

(c)

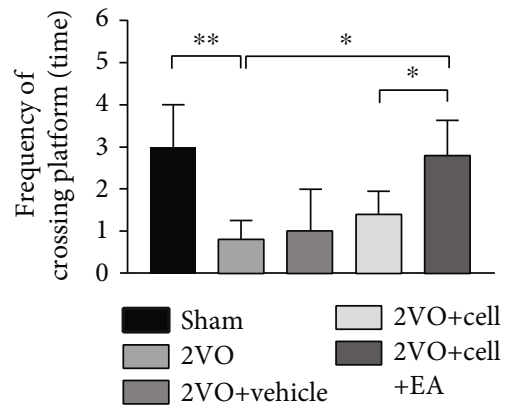

(d)

Figure 8: Comparison of the effects on spatial learning and memory test in rats from different groups. (a) (A-E) The swimming track of different groups of rats in spatial learning training; (F-J) the swimming track of different groups of rats in the spatial memory test. (A) and (F) from the sham group; (B) and (G) from the 2VO group; (C) and (H) from the 2VO+Vehicle group; (D) and (I) from the 2VO + Cell group; (E) and (J) from the 2VO+Cell+EA group. (b) Comparison of the average escape latencies of five groups in four days of spatial learning training (one-way ANOVA, $F=12.354, P<0.001$ ). (c) Comparison of the swimming time in target quadrant of five groups in the spatial memory test (one-way ANOVA, $F=22.159, P<0.001$ ). (d) Comparison of the frequency of crossing in the platform of five groups in the spatial memory test (one-way ANOVA, $F=8.281, P<0.01)$. Values are mean \pm SEM $(N=5$ rats/per group). ${ }^{*} P<0.05,{ }^{* *} P<0.01$, and ${ }^{* * *} P<0.001$.

surviving cells coexpressing $\mathrm{HuNu}$ (for analysis of the survival of grafted cells, all nuclei of grafted cells were identified based on $\mathrm{HuNu}$ immunostaining) and mCherry were observed. These cells also expressed NeuN (fluorescence intensity was not as strong as the host cells), suggesting that they were gradually differentiating toward mature neurons. More $\mathrm{HuNu}^{+}$cells were detected in the $2 \mathrm{VO}+\mathrm{Cell}+\mathrm{EA}$ group compared with the $2 \mathrm{VO}+\mathrm{Cell}$ group through cell count analysis. Considering the above research results, we speculated that the higher survival of grafted cells in the $2 \mathrm{VO}+\mathrm{Cell}+\mathrm{EA}$ group was related to the EA effect on suppressing inflammatory and promoting angiogenesis.

As already established, the hippocampus plays a critical role in cognitive function [38]. As the basic structure or constitution, neurons of the hippocampus serve its local circuitry and process information. Since the loss of hippocampal neurons would induce cognitive deficits [39], cerebral ischemia could lead to selective and delayed pyramidal neuronal death in the hippocampal CA 1 area [40]. Thus, replenishing lost hippocampal neurons after ischemia may become an important treatment strategy to reduce cognitive damage. In the MWM task, compared with the rats in the $2 \mathrm{VO}$ group, the rats in the $2 \mathrm{VO}+\mathrm{Cell}$ group showed a reduction in the escape latency and increased time spent in the target quadrant, indicating that learning and memory impairment in $2 \mathrm{VO}$ rats was alleviated. We speculated that the improved cognitive function was related to the replacement of partial dead hippocampal neurons by MGE neural progenitor transplantation. Compared with the $2 \mathrm{VO}+\mathrm{Cell}$ group, the rats in the 2VO+Cell+EA group showed increased time spent in the target quadrant, and the frequency of crossing the platform was increased. This indicated that the ability of rats to learn and memorize in the $2 \mathrm{VO}+\mathrm{Cell}+\mathrm{EA}$ group was improved more significantly. Considering that more $\mathrm{HuNu}^{+}$-positive cells were observed in the 2VO+Cell+EA group in cellular quantification analysis, we speculated that EA facilitated the cell grafting effect on learning and memory impairment by promoting the grafted cells' survival in the hippocampus.

In summary, our study demonstrated that EA could promote the survival of the grafted MGE neural progenitors differentiated from human embryonic pluripotent stem cells and alleviated learning and memory impairment in rats with cerebral ischemia, which partially resulted from inhibited inflammation response and increased angiogenesis. This observation indicates that the combination of MGE neural progenitors with EA might be a promising approach to treat cognitive deficits after cerebral infarct. However, further 
studies and long-term observations are required to explore whether the grafted cells can differentiate into cholinergic and GABAergic neurons after maturation, their interaction and integration with host cells, and the formation of synaptic circuits to alleviate neural function deficits.

\section{Data Availability}

The data used to support the findings of this study are available from the corresponding author upon reasonable request.

\section{Conflicts of Interest}

The authors have declared no conflict of interest.

\section{Authors' Contributions}

Xiaohua Han and Hong Chen conceived and designed the project. Juan Li, Luting Chen, and Danping Li carried out the experiment. Min Lu and Xiaolin Huang analyzed the data. Juan Li, Luting Chen, and Xiaohua Han drafted the manuscript. All authors read and approved the final manuscript. Juan Li and Luting Chen contributed equally to this work.

\section{Acknowledgments}

This study was supported by the National Natural Science Foundation of China (Grant No. 81774404). Thanks are due to Professor Su-chun Zhang from Singapore DukeNUS for kindly providing the H9 human embryonic stem cell line and the chemically modified H9-mCherry human embryonic stem cell line.

\section{References}

[1] D. Mozaffarian, E. J. Benjamin, A. S. Go et al., "Heart disease and stroke statistics-2016 update: a report from the American Heart Association," Circulation, vol. 133, no. 4, pp. e38-e360, 2016.

[2] A. Kiryk, R. Pluta, I. Figiel et al., "Transient brain ischemia due to cardiac arrest causes irreversible long- lasting cognitive injury," Behavioural Brain Research, vol. 219, no. 1, pp. 1-7, 2011.

[3] M. Gulinello, D. Lebesgue, T. Jover-Mengual, R. S. Zukin, and A. M. Etgen, "Acute and chronic estradiol treatments reduce memory deficits induced by transient global ischemia in female rats," Hormones and Behavior, vol. 49, no. 2, pp. 246260, 2006.

[4] J. Emberson, K. R. Lees, P. Lyden et al., "Effect of treatment delay, age, and stroke severity on the effects of intravenous thrombolysis with alteplase for acute ischaemic stroke: a metaanalysis of individual patient data from randomised trials," Lancet, vol. 384, no. 9958, pp. 1929-1935, 2014.

[5] W. J. Powers, A. A. Rabinstein, T. Ackerson et al., "2018 guidelines for the early management of patients with acute ischemic stroke: a guideline for healthcare professionals from the American Heart Association/American Stroke Association," Stroke, vol. 49, no. 3, pp. e46-e110, 2018.
[6] D. L. Rimmele and G. Thomalla, "Wake-up stroke: clinical characteristics, imaging findings, and treatment option â€ an update," Frontiers in Neurology, vol. 5, no. 35, 2014.

[7] C. Xie, X. Gao, Y. Luo, Y. Pang, and M. Li, "Electroacupuncture modulates stromal cell-derived factor- $1 \alpha$ expression and mobilization of bone marrow endothelial progenitor cells in focal cerebral ischemia/reperfusion model rats," Brain Research, vol. 1648, no. Part A, pp. 119-126, 2016.

[8] S. Palma-Tortosa, D. Tornero, M. G. Hansen et al., "Activity in grafted human iPS cell-derived cortical neurons integrated in stroke-injured rat brain regulates motor behavior," Proceedings of the National Academy of Sciences of the United States of America, vol. 117, no. 16, pp. 9094-9100, 2020.

[9] E. Curtis, J. R. Martin, B. Gabel et al., “A first-in-human, phase I study of neural stem cell transplantation for chronic spinal cord injury," Cell Stem Cell, vol. 22, no. 6, pp. 941-950.e6, 2018.

[10] S. Gao, X. Guo, S. Zhao et al., "Differentiation of human adipose-derived stem cells into neuron/motoneuron- like cells for cell replacement therapy of spinal cord injury," Cell Death \& Disease, vol. 10, no. 8, p. 597, 2019.

[11] L. Zhao, C. Zhou, L. Li et al., "Acupuncture improves cerebral microenvironment in mice with Alzheimer's disease treated with hippocampal neural stem cells," Molecular Neurobiology, vol. 54, no. 7, pp. 5120-5130, 2017.

[12] L. Magno, C. Barry, C. Schmidt-Hieber, P. Theodotou, M. Häusser, and N. Kessaris, "NKX2-1 is required in the embryonic septum for cholinergic system development, learning, and memory," Cell Reports, vol. 20, no. 7, pp. 1572-1584, 2017.

[13] M. Emgård, U. Hallin, J. Karlsson, B. A. Bahr, P. Brundin, and K. Blomgren, "Both apoptosis and necrosis occur early after intracerebral grafting of ventral mesencephalic tissue: a role for protease activation," Journal of Neurochemistry, vol. 86, no. 5, pp. 1223-1232, 2003.

[14] C. E. Sortwell, M. R. Pitzer, and T. J. Collier, "Time course of apoptotic cell death within mesencephalic cell suspension grafts: implications for improving grafted dopamine neuron survival," Experimental Neurology, vol. 165, no. 2, pp. 268 $277,2000$.

[15] M. Uemura, M. M. Refaat, M. Shinoyama et al., "Matrigel supports survival and neuronal differentiation of grafted embryonic stem cell-derived neural precursor cells," Journal of Neuroscience Research, vol. 88, no. 3, pp. 542-551, 2010.

[16] W. M. Zawada, D. J. Zastrow, E. D. Clarkson, F. S. Adams, K. P. Bell, and C. R. Freed, "Growth factors improve immediate survival of embryonic dopamine neurons after transplantation into rats," Brain Research, vol. 786, no. 1-2, pp. 96-103, 1998.

[17] M.-h. Shen, C.-b. Zhang, J.-h. Zhang, and P.-f. Li, "Electroacupuncture attenuates cerebral ischemia and reperfusion injury in middle cerebral artery occlusion of rat via modulation of apoptosis, inflammation, oxidative stress, and excitotoxicity," Evidence-Based Complementary and Alternative Medicine, vol. 2016, Article ID 9438650, 2016.

[18] X. Han, X. Zhao, and M. Lu, "Electroacupuncture ameliorates learning and memory via activation of the CREB signaling pathway in the hippocampus to attenuate apoptosis after cerebral hypoperfusion," Evidence-based complementary and alternative medicine, vol. 2013, Article ID 156489, 2013.

[19] S. J. Wang, N. Omori, G. Jin et al., "Functional improvement by electro-acupuncture after transient middle cerebral artery 
occlusion in rats," Neurological Research, vol. 25, no. 5, pp. 516-521, 2003.

[20] L. Lu, X. G. Zhang, and L. L. Zhong, “Acupuncture for neurogenesis in experimental ischemic stroke: a systematic review and meta-analysis," Scientific Reports, vol. 6, 2016.

[21] H. Jin, Y.-T. Zhang, Y. Yang et al., "Electroacupuncture facilitates the integration of neural stem cell-derived neural network with transected rat spinal cord," Stem Cell Reports, vol. 12, no. 2, pp. 274-289, 2019.

[22] Y. R. Kim, S. M. Ahn, M. E. Pak et al., "Potential benefits of mesenchymal stem cells and electroacupuncture on the trophic factors associated with neurogenesis in mice with ischemic stroke," Scientific Reports, vol. 8, no. 1, 2018.

[23] S. M. Chambers, C. A. Fasano, E. P. Papapetrou, M. Tomishima, M. Sadelain, and L. Studer, "Highly efficient neural conversion of human ES and iPS cells by dual inhibition of SMAD signaling," Nature Biotechnology, vol. 27, no. 3, pp. 275-280, 2009.

[24] C. X. Zheng, M. Lu, and Y. B. Guo, "Electroacupuncture ameliorates learning and memory and improves synaptic plasticity via activation of the PKA/CREB signaling pathway in cerebral hypoperfusion," Evidence-Based Complementary and Alternative Medicine, vol. 2016, Article ID 7893710, 2016.

[25] J.-J. Peng, R. Sha, M.-X. Li et al., "Repetitive transcranial magnetic stimulation promotes functional recovery and differentiation of human neural stem cells in rats after ischemic stroke," Experimental Neurology, vol. 313, pp. 1-9, 2019.

[26] G. Paxinos and C. Watson, The Rat Brain in Stereotaxic Coordinates, Academic Press, New York, 2008.

[27] Y. Liu, J. P. Weick, H. Liu et al., "Medial ganglionic eminencelike cells derived from human embryonic stem cells correct learning and memory deficits," Nature Biotechnology, vol. 31, no. 5, pp. 440-447, 2013.

[28] J. Wang, X. Fu, C. Jiang et al., "Bone marrow mononuclear cell transplantation promotes therapeutic angiogenesis via upregulation of the VEGF-VEGFR2 signaling pathway in a rat model of vascular dementia," Behavioural Brain Research, vol. 265, no. 2014, pp. 171-180, 2014.

[29] D. Yang, Z. J. Zhang, and M. Oldenburg, "Human embryonic stem cell-derived dopaminergic neurons reverse functional deficit in parkinsonian rats," Stem Cells (Dayton, Ohio), vol. 26 , no. 1 , pp. 55-63, 2008.

[30] J. Anrather and C. Iade, "Inflammation and stroke: an overview," Neurotherapeutics, vol. 13, no. 4, pp. 661-670, 2016.

[31] J. Miyanohara, M. Kakae, K. Nagayasu et al., "TRPM2 channel aggravates CNS inflammation and cognitive impairment via activation of microglia in chronic cerebral hypoperfusion," The Journal of Neuroscience, vol. 38, no. 14, pp. 3520-3533, 2018.

[32] A. S. Chung and N. Ferrara, "Developmental and pathological angiogenesis," Annual Review of Cell and Developmental Biology, vol. 27, no. 1, pp. 563-584, 2011.

[33] E.-h. Chung, K. Iwasaki, K. Mishima, N. Egashira, and M. Fujiwara, "Repeated cerebral ischemia induced hippocampal cell death and impairments of spatial cognition in the rat," Life Sciences, vol. 72, no. 4-5, pp. 609-619, 2002.

[34] H. J. Park, H. S. Shim, K. S. Kim, and I. Shim, "The protective effect of black ginseng against transient focal ischemia-induced neuronal damage in rats," The Korean Journal of Physiology \& Pharmacology, vol. 15, no. 6, pp. 333-338, 2011.
[35] Y. Tao and S. C. Zhang, "Neural subtype specification from human pluripotent stem cells," Cell Stem Cell, vol. 19, no. 5, pp. 573-586, 2016.

[36] X. Han, H. Jin, K. Li et al., "Acupuncture modulates disrupted whole-brain network after ischemic stroke: evidence based on graph theory analysis," Neural Plasticity, vol. 2020, Article ID 8838498, 10 pages, 2020.

[37] H. Liu, L. Chen, G. Zhang et al., "Scalp acupuncture enhances the functional connectivity of visual and cognitive-motor function network of patients with acute ischemic stroke," Evidence-Based Complementary and Alternative Medicine, vol. 2020, Article ID 8836794, 11 pages, 2020.

[38] R. A. Epstein, E. Z. Patai, J. B. Julian, and H. J. Spiers, "The cognitive map in humans: spatial navigation and beyond," Nature Neuroscience, vol. 20, no. 11, pp. 1504-1513, 2017.

[39] G. Li, H. Cheng, X. Zhang et al., "Hippocampal neuron loss is correlated with cognitive deficits in SAMP8 mice," Neurological Sciences, vol. 34, no. 6, pp. 963-969, 2013.

[40] L. Kamali Dolatabadi, M. Emamghoreishi, M. R. Namavar, and H. Badeli Sarkala, "Curcumin effects on memory impairment and restoration of irregular neuronal distribution in the hippocampal CA1 region after global cerebral ischemia in male rats," Basic and Clinical Neuroscience, vol. 10, no. 5, pp. 527-539, 2019. 